\title{
Ablation of FGFR2 in Fibroblasts Ameliorates Kidney Fibrosis after Ischemia/Reperfusion Injury in Mice
}

\author{
Zhuo Xu Chunsun Dai
}

Center for Kidney Disease, Second Affiliated Hospital, Nanjing Medical University, Nanjing, China

\section{Keywords}

FGFR2 · Fibroblast · Fibrosis · Kidney · Signaling

transduction

\begin{abstract}
Background: Fibroblast growth factors (FGFs) are heparinbinding proteins involved in a variety of biological processes. However, the role and mechanisms of FGF/FGFR2 signaling in fibroblast activation and kidney fibrosis need further investigation. Methods: In this study, a mouse model with fibroblast-specific FGFR2 gene disruption was generated. The knockouts were born normal and no kidney dysfunction or histological abnormality was found within 2 months after birth. A kidney ischemia/reperfusion injury (IRI) model was created. Results: Kidney fibrosis was developed in the control littermates within 2 and 4 weeks after IRI, while in the knockouts, total collagen deposition, fibronectin, and alpha smooth muscle actin expression were decreased compared to those in the control littermates. In addition, the numbers of Ki-67-positive interstitial cells as well as TUNEL-positive interstitial cells were lower in the knockout kidneys at 4 weeks after IRI. Phosphorylated extracellular regulated protein kinase 1/2 was decreased in the knockout kidneys at 2 and 4 weeks after IRI compared to those in the control lit-
\end{abstract}

\section{KARGER}

(C) 2017 S. Karger AG, Basel termates. Conclusion: These results suggest that FGF/FGFR2 signaling may promote the proliferation and activation of kidney fibroblasts, which contribute to the development of kidney fibrosis.

(c) 2017 S. Karger AG, Basel

\section{Introduction}

About $10 \%$ of the population worldwide suffer from chronic kidney diseases [1-4]. Regardless of the etiology of the primary disease, kidney fibrosis is one of the most common pathologic characteristics and finally leads to end-stage renal disease. It has been well demonstrated that myofibroblasts play a determining role in initiating and promoting kidney fibrosis. Despite many cell types, such as tubular cells, endothelial cells, and fibroblasts contributing to kidney fibrosis, resident fibroblasts transformed to myofibroblasts are the major source of kidney interstitial extracellular matrix (ECM) production [5]. In normal adult kidneys, fibroblasts are usually quiescent in the interstitium between the capillaries and the tubular epithelium. After stimulation, fibroblasts acquire a myofibroblast phenotype by expressing alpha smooth muscle actin ( $\alpha$-SMA) and producing a large amount of ECM 
components, such as fibronectin (FN), type I collagen, type IV collagen, and proteoglycans [6-10]. Several profibrotic factors, including transforming growth factor $\beta 1$, platelet-derived growth factor, and connective tissue growth factor, are able to promote fibroblast activation and proliferation [11-14]. However, the role and mechanisms of the other growth factors, such as fibroblast growth factors (FGFs), in regulating fibroblast activation and kidney fibrosis need more investigation.

FGFs are a group of heparin-binding proteins involved in many biological processes, such as embryonic development, tumorigenesis, cell proliferation, differentiation, as well as cell survival $[15,16]$. Phylogenetic analysis of the Fgf gene family identifies seven subfamilies, which may be divided into intracrine, paracrine, and endocrine FGFs. Except for intracrine FGFs, which are not secreted, most FGFs mediate biological responses by binding to their cell surface receptors (FGFRs). In most cell types, binding of FGFs with FGFRs may activate intracellular signaling pathways, including JNK/STAT signal, MAP kinase, PI3K/AKT, and phospholipase $C \gamma$ [17-19]. In developing mammalian kidneys, all four types of FGFR and several FGFs, such as FGF2, FGF7, FGF8, and FGF10, can be detected [20-23]. A series of studies using genetic animal models showed that FGF/FGFR signaling plays a key role for renal development. For example, mice null for FGF7 or FGF10 exhibit small kidneys and few branches of the ureteric bud [24-26]. Conditional deletion of FGF8 from the metanephric mesenchyme interrupts nephron formation in mice [27]. Specific deletion of both FGFR1 and FGFR2 in the metanephric mesenchyme results in small kidneys, fewer nephrons, as well as an unbranched ureteric bud. Deleting FGFR2 in renal and urinary tract mesenchyme leads to ureteric bud induction defection and displaced ureteric buds along the wolffian duct or duplex ureters as well as vesicoureteral reflux [28]. As previously reported, long-term (8-13 weeks) FGF2 treatment may result in focal segmental glomerulosclerosis in adult rats $[29,30]$. In mice with unilateral ureteral obstruction, infusion of FGF7 resembles the urothelium on day 14 following obstruction by causing the formation of a bladder-like multistratified barrier with enhanced apical uroplakin plaques [31]. However, the role and mechanisms of FGF/FGFR2 signaling in fibroblast activation and kidney fibrosis are not fully known.

In this study, we found that mice with fibroblast-specific deletion of FGFR2 were resistant to kidney fibrosis at 1 month after ischemia/reperfusion injury (IRI). Interstitial cell proliferation as well as extracellular regulated protein kinase 1/2 (Erk1/2) phosphorylation in the kid- neys from the knockouts were also diminished at 4 weeks after IRI. This study suggests that FGF/FGFR2 signaling may play an important role for fibroblast activation and kidney fibrosis.

\section{Methods}

Mice and Animal Model

FGFR2 floxed mice and S100A4-Cre transgenic mice were purchased from Jackson Laboratory [32]. By mating the FGFR2 floxed mice with S100A4-Cre transgenic mice, mice heterozygous for the FGFR2 floxed alleles (genotype: FGFR2 fl/wt, Cre+/-) were generated. In order to inactivate both FGFR alleles, these mice were crossbred. We then obtained conditional knockout mice in which the FGFR2 gene was specifically disrupted in fibroblasts (genotype: FGFR2 fl/fl, Cre+/-). The breeding protocol also generated heterozygous littermates (genotype: FGFR2 fl/wt, $\mathrm{Cre}+/-$ ) and wild-type and several groups with different genotypes (FGFR2 fl/fl, Cre-l-, or FGFR2 fl/wt, Cre-/-). Mice were identified using a PCR reaction with genomic DNA isolated from tail biopsy within 3 weeks after birth. The primers used for genotyping were as follows: Cre sense $5^{\prime}$-GCA GAT CTG GCT CTC CAA AG-3', Cre antisense 5' AGG CAA ATT TTG GTG TAC GG-3'; FGFR2 sense $5^{\prime}$-TTC CTG TTC GAC TAT AGG AGC AAC AGG CGG-3', FGFR2 antisense $5^{\prime}$-GAG AGC AGG GTG CAA GAG GCG ACC AGT CAG- $3^{\prime}$. All mice with an age ranging from 6 to 8 weeks were used in the study. Littermates with genotype (FGFR2 fl/fl, Cre-/-) were used as controls.

For the unilateral kidney IRI model, S100A4-FGFR2-/- animals and their control littermates were anesthetized as mentioned above, then the kidneys were exposed through a midline incision, and the left renal pedicle was clamped for $40 \mathrm{~min}$. Mice were kept in the incubator at $38^{\circ} \mathrm{C}$, and the abdominal cavity was hydrated with saline-moistened gauze. Following clamp removal, the kidney was visually assessed for reperfusion within $1 \mathrm{~min}$. Mice in the sham control group were prepared as above but without clamping of the left renal pedicle. After surgery, the animals were returned to their cages and allowed free access to food and water. Mice were sacrificed at 14 and 28 days after reperfusion. To determine the role of the deletion of FGFR2 in fibroblasts on tubular damage and acute kidney injury, in some experiments the right kidney was ablated immediately after the left kidney reperfusion, and blood was collected on days 1, 2, 4, and 7. Blood urea nitrogen (BUN) and serum creatinine were measured.

Kidney tissues were harvested and snap-frozen in liquid nitrogen, then stored at $-80^{\circ} \mathrm{C}$ for extraction of RNA and protein. For histological evaluation, kidneys were fixed in $10 \%$ phosphate-buffered formalin followed by paraffin embedding.

Urinary Albumin, Creatinine, lysozyme, and N-Acetyl- $\beta-D-$ Glucosaminidase Assay

Urinary albumin concentration was measured by using a mouse Albumin ELISA Quantification kit (Bethyl Laboratories, Montgomery, TX, USA). Urinary creatinine was determined by using a creatinine Assay kit (DICT-500; BioAssay System, Hayward, CA, USA). Urinary $\mathrm{N}$-acetyl- $\beta$-D-glucosaminidase (NAG) was detected by using the NAG assay kit (Nanjing Jiancheng Bioengineering, Nanjing, China). Urinary lysozyme was detected by using Micro-

FGFR2 in Kidney Fibrosis

Kidney Dis 2017;3:160-170 
coccus luteus as its substrate. Briefly, $20 \mu \mathrm{L}$ of urine or $\mathrm{ddH}_{2} \mathrm{O}$ were incubated at $37^{\circ} \mathrm{C}$ for 3 min with $200 \mu \mathrm{L}$ of the substrate solution at a concentration of $100 \mu \mathrm{g} / \mathrm{mL}$ at $\mathrm{pH} 6.4$, then the absorbance was read at $450 \mathrm{~nm}$, and the lysozyme in urine $(\mathrm{mg} / \mathrm{dL})$ was calculated.

\section{Detection of Serum BUN and Creatinine}

Serum BUN and creatinine levels were measured by using a mouse BUN ELISA Quantification kit (Cat: DIUR-500; BioAssay System) and creatinine assay kit (mentioned above) respectively, according to the manufacturer's instruction.

\section{Western Blot Analysis}

Protein from kidney tissue was extracted with RIPA buffer composed of $1 \times$ PBS, $1 \%$ NP-40, $0.1 \%$ SDS, $0.1 \%$ sodium deoxycholate, $1 \mu \mathrm{M}$ sodium orthovanadate, $100 \mathrm{nM}$ PMSF, $1 \%$ protease inhibitor cocktail (Cat: P8340; Sigma-Aldrich, St. Louis, MO, USA), and 1\% phosphatase II and III inhibitor cocktail (Cat: P5726 and P0044, respectively; Sigma-Aldrich) on ice. The supernatants were collected after centrifugation at $16,000 \mathrm{~g}$ for $30 \mathrm{~min}$ at $4^{\circ} \mathrm{C}$. Protein concentration was measured using the BCA assay (Pierce), and an equal amount of protein $(25-50 \mu \mathrm{g})$ was separated on $8-12 \%$ tris-glycine polyacrylamide gels. After transfer onto nitrocellulose membranes, the target protein was detected by using a specific primary antibody. The primary antibodies used were as follows: anti-FN (Cat: F3648; Sigma-Aldrich), anti-a-SMA (Cat: A2547; Sigma-Aldrich), antip-Erk1/2 (Thr202/Tyr204) (Cat: 4370; Cell Signaling Technology, Beverly, MA, USA), anti-Erk1/2 (Cat: 4695; Cell Signaling Technology), and anti-GAPDH (cat: FL-335; Santa Cruz Biotechnology, Santa Cruz, CA, USA). Quantification was performed by measurement of the intensity of the signals with the Image J software.

\section{Immunohistochemistry and Immunofluorescence Staining}

Kidney tissues were fixed in $4 \%$ neutral formalin at room temperature, followed by embedment in paraffin. Then the tissue was cut into $2-\mu \mathrm{m}$-thick slices, deparaffinized in xylene, hydrated, and stained with H\&E, PAS, and Sirius Red. In some experiments, tissue sections were immunostained with primary antibodies against anti-FGFR2 (Cat: sc-122; Santa Cruz Biotechnology). For immunofluorescence staining, kidney cryosections were fixed with $3.7 \%$ paraformaldehyde for $15 \mathrm{~min}$ at room temperature and immersed in $0.2 \%$ Triton X-100 for 10 min. After blocking with $10 \%$ donkey serum in PBS for $1 \mathrm{~h}$, slides were immunostained with the following antibodies: anti-FN, anti-a-SMA (Cat: A2547; Sigma-Aldrich), and anti-p-Erk1/2 (Thr202/Tyr204). The slides were viewed with a Nikon Eclipse 80i Epi-fluorescence microscope equipped with a digital camera (DS-Ri1; Nikon).

\section{Quantitative Determination of Total Collagen in Kidney}

Tissue

The kidney tissue collagen was quantitated as previously reported $[14,33]$. Briefly, $4-\mu$ m-thick sections of paraffin-embedded tissue were deparaffinized in xylene, hydrated, and then covered with $200 \mu \mathrm{L}$ dye solution $(0.1 \%$ Sirius Red and $0.1 \%$ Fast Green dissolved in water-saturated picric acid) overnight at room temperature. After washing three times with $\mathrm{ddH}_{2} \mathrm{O}$, the dye was eluted from tissue sections with a mixture of $0.1 \mathrm{~N} \mathrm{NaOH}$ and methanol (1:1). Absorbances at 540 and $605 \mathrm{~nm}$ were determined for Sirius Red-binding collagen and Fast Green-binding protein, respectively. This result was presented as microgram collagen per milligram of total protein.

\section{TUNEL Staining}

Apoptotic cell death was determined by using a TUNEL staining system (Promega, Madison, WI, USA), and the cells staining positive were counted.

\section{Statistical Analysis}

The experimental data are presented as mean \pm SEM. Statistical analysis of the data was performed using the SPASS 13.0 software. Comparison among groups was made by one-way ANOVA, followed by post hoc Student-Newman-Keuls method or post hoc least significant difference. The Student $t$ test was used for the comparison between the two groups. $p<0.05$ was considered statistically significant.

\section{Results}

\section{Mice with Fibroblast Ablation of FGFR2 Were \\ Phenotypically Normal}

To investigate the physiological role of FGFR2 in fibroblast activation and renal fibrosis, we generated a mouse model with FGFR2 gene-specific deletion in fibroblasts by using the Cre-LoxP system. As shown in Figure 1a, mice with fibroblast-specific ablation of FGFR2 was designated as S100A4-FGFR2-/- (genotype: FGFR2 fl/fl, Cre +/-, lane 3), whereas the littermates with the same sex were used as control (genotype: FGFR2 fl/fl, Cre-/-, lane 1).

Immunohistochemical staining showed that the FGFR2 protein level was reduced in interstitial cells from S100A4-FGFR2-/- mice compared to those from control littermates on day 28 after IRI (Fig. 1b). S100A4FGFR2-/- mice are viable, fertile, and have a normal lifespan. There was no difference in the body weight of both sexes or the ratio of kidney/body weight 8 weeks after birth between the two groups (Fig. 1c, f). Kidney function parameters, including serum creatinine, BUN, urinary NAG, urinary lysozyme, and urinary albumin level, were comparable between S100A4-FGFR2-/- mice and their control littermates at 8 weeks after birth (Fig. 1d, e, g-i). H\&E and PAS staining showed no obvious kidney morphologic change between S100A4-FGFR2-/- mice and their control littermates (Fig. 1j). These results demonstrate that specific deletion of FGFR2 in fibroblasts does not affect kidney function or histology under physiological condition within 2 months after birth.

\section{Mice with Fibroblast Ablation of FGFR2 Were \\ Resistant to Kidney Fibrosis after IRI}

To investigate the role of FGF/FGFR2 signaling in renal fibrosis, we challenged the mice with IRI to induce kidney injury, and the kidneys were harvested on days 14 and 28 after surgery. Acute kidney injury was induced at
$\mathrm{Xu} / \mathrm{Dai}$ 


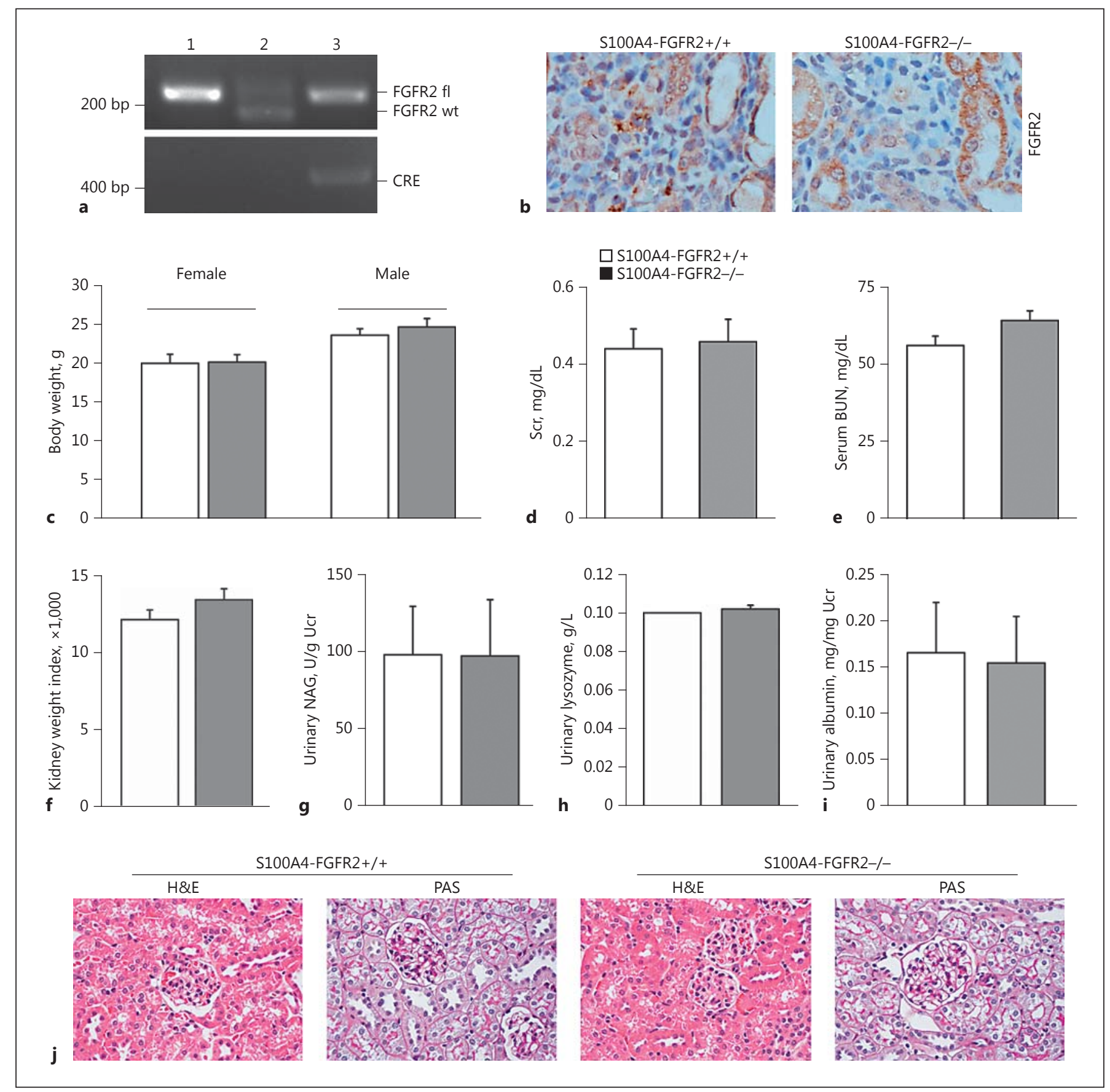

Fig. 1. Generation of the mice with fibroblast-specific deletion of FGFR2. a Genotyping of the mice by PCR assay of genomic DNA. Lane 1: genotype FGFR2 fl/fl, Cre-/-; lane 2: genotype FGFR2 $\mathrm{fl} /$ wt, Cre-/-; lane 3: genotype FGFR2 fl/fl, Cre+/-, designated as S100A4-FGFR2-/-. b Representative immunohistochemical staining images showing the loss of FGFR2 in interstitial cells from the fibrotic kidneys in S100A4-FGFR2-/- mice. c-i Mice with fibroblast-specific ablation of FGFR2 exhibited little difference in body weight (c), serum creatinine (d), serum BUN (e), kidney weight index (f), urinary NAG (g), urinary lysozyme (h), and urinary albumin (i) levels compared to their control littermates within 8 weeks after birth $(n=3)$. $\mathbf{j}$ Representative images for PAS and $\mathrm{H} \& \mathrm{E}$ staining showing no obvious kidney morphological abnormality in S100A4-FGFR2-/- mice at 8 weeks after birth. BUN, blood urea nitrogen; FGFR, fibroblast growth factor receptor; NAG, $\mathrm{N}$-acetyl- $\beta$-D-glucosaminidase; Scr, serum creatinine; Ucr, urinary creatinine. 


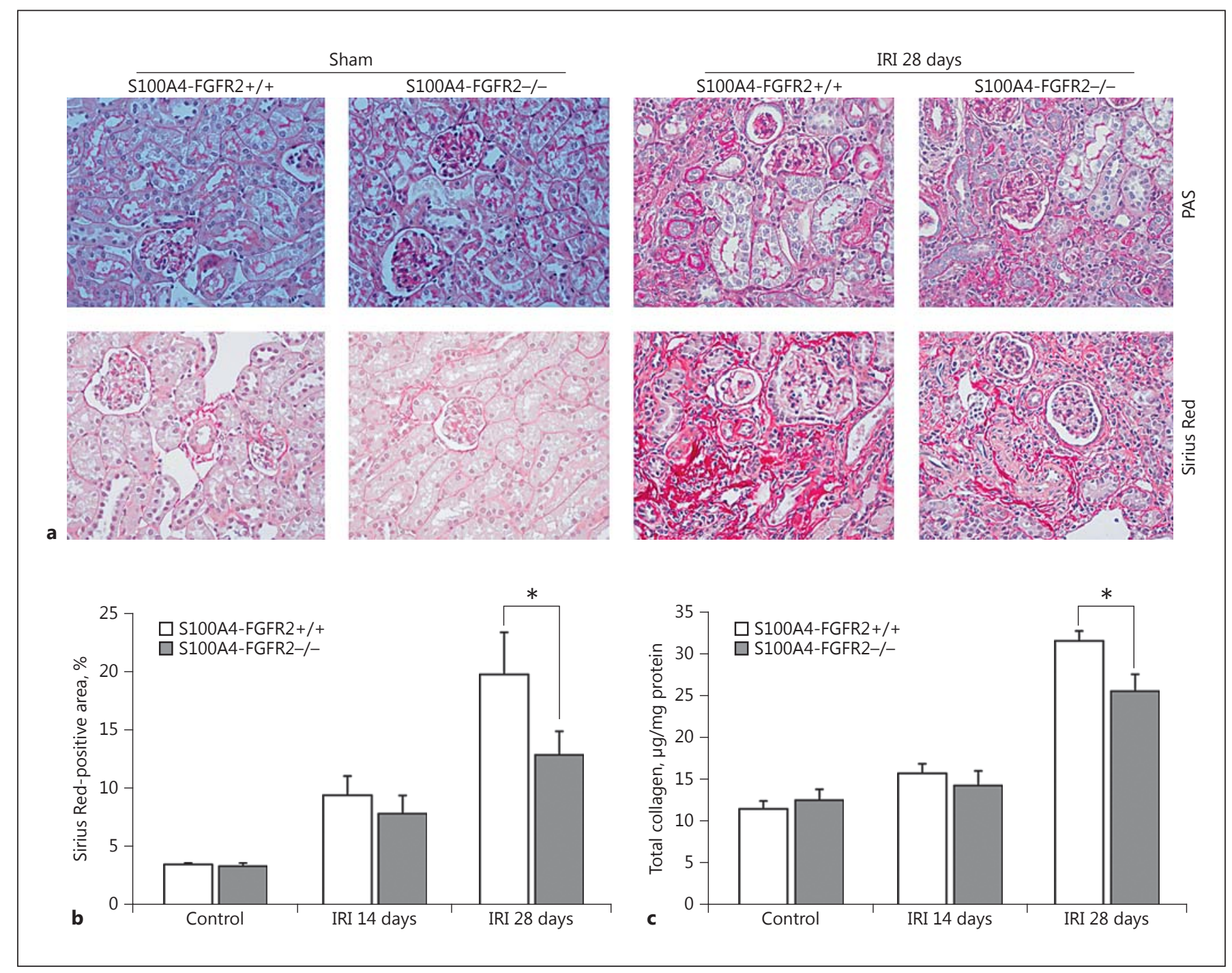

Fig. 2. Deletion of FGFR2 in fibroblasts ameliorated kidney fibrosis after IRI. a Representative images for PAS and Sirius Red staining in sham and IRI kidneys from S100A4-FGFR2-/- mice and control littermates. b Graph showing quantitative results for the area staining positive for Sirius Red. ${ }^{*} p<0.05$ versus control lit-

$24 \mathrm{~h}$ after IRI, reflected as increased BUN and serum creatinine in both the knockouts and their control littermates, but no difference was found between the two groups (data not shown), suggesting that fibroblast-specific deletion of FGFR2 does not affect IRI-induced acute kidney injury. In control littermates, at 28 days after surgery, chronic kidney damage reflected as loss of brush border, tubular cell atrophy, thickening and shrinkage of tubular basement membrane, and development of interstitial fibrosis were less severe in the knockout kidneys

termates, $n=6$. c Graph showing the results for total collagen content in kidneys from S100A4-FGFR2-/- mice and control littermates. ${ }^{*} p<0.05$ versus control littermates, $n=6$. FGFR, fibroblast growth factor receptor; IRI, ischemia/reperfusion injury.

(Fig. 2a). The area staining positive for Sirius Red in the knockout kidneys was significantly decreased at 28 days after IRI compared to that in the control littermates (Fig. 2a, b). Similarly, quantitative determination of total collagen in kidney tissues showed a significant reduction in S100A4-FGFR2-/- mice compared to control mice at 28 days after IRI (Fig. 2c). Therefore, the above data suggest that renal fibrosis developing after IRI was markedly ameliorated in mice with fibroblast-specific FGFR2 gene deletion. 


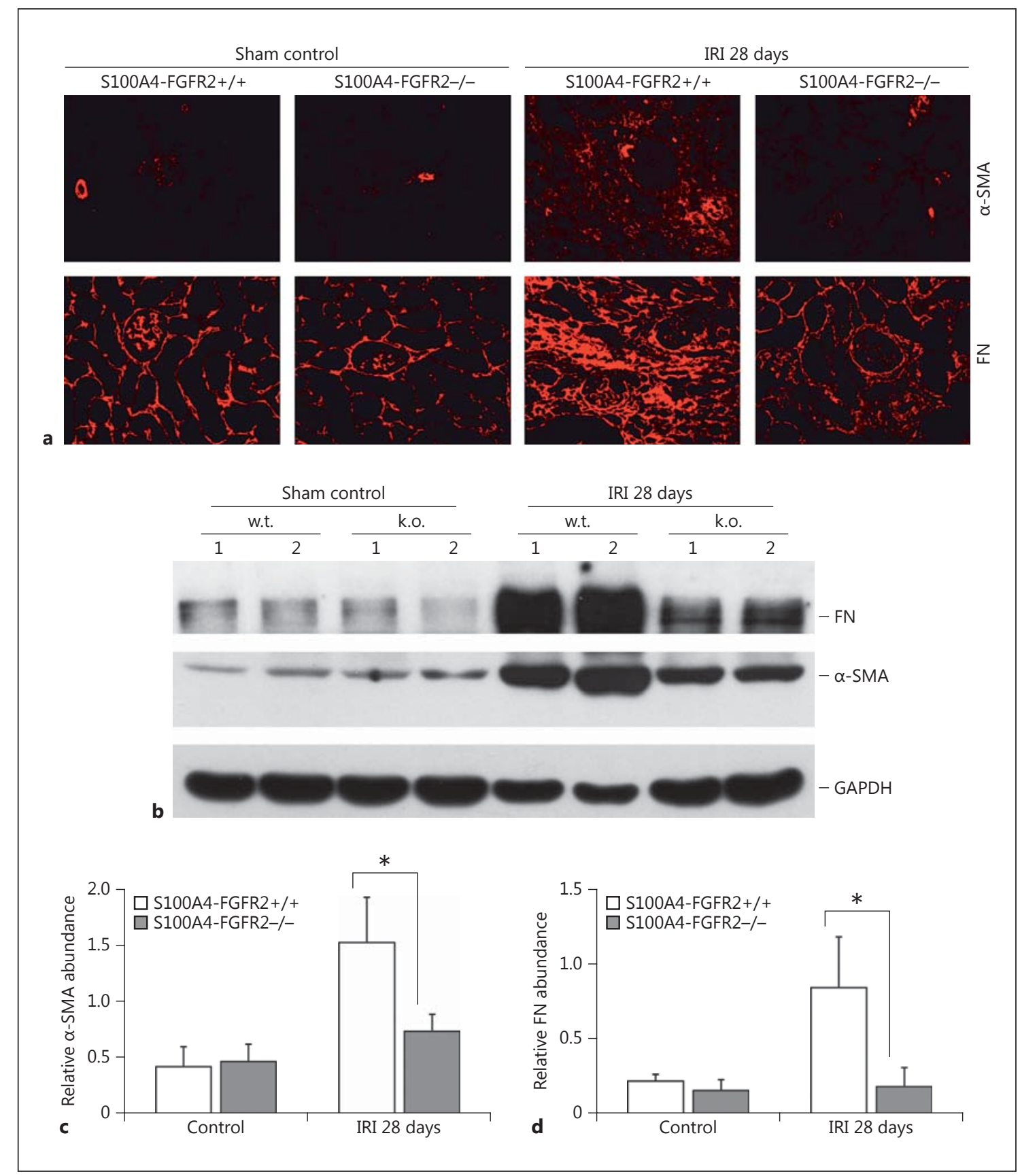

Fig. 3. Deletion of FGFR2 in fibroblasts inhibits FN and a-SMA expression in the kidneys after IRI. a Representative images for FN and $a$-SMA immunostaining in the kidneys from S100A4FGFR2-/- mice and control littermates. b Western blot assay showing the abundance of FN and $a-S M A$ in the kidneys from S100A4-FGFR2-/- mice and control littermates. Numbers indi- cate individual animals. The gels were run under the same experimental conditions. $\mathbf{c}$, d Graphs showing the semiquantitative results for the abundance of $\alpha$-SMA (c) and FN (d). ${ }^{*} p<0.05$ versus control littermates, $n=4$. $\alpha$-SMA, alpha smooth muscle actin; FGFR, fibroblast growth factor receptor; FN, fibronectin; IRI, ischemia/reperfusion injury. 


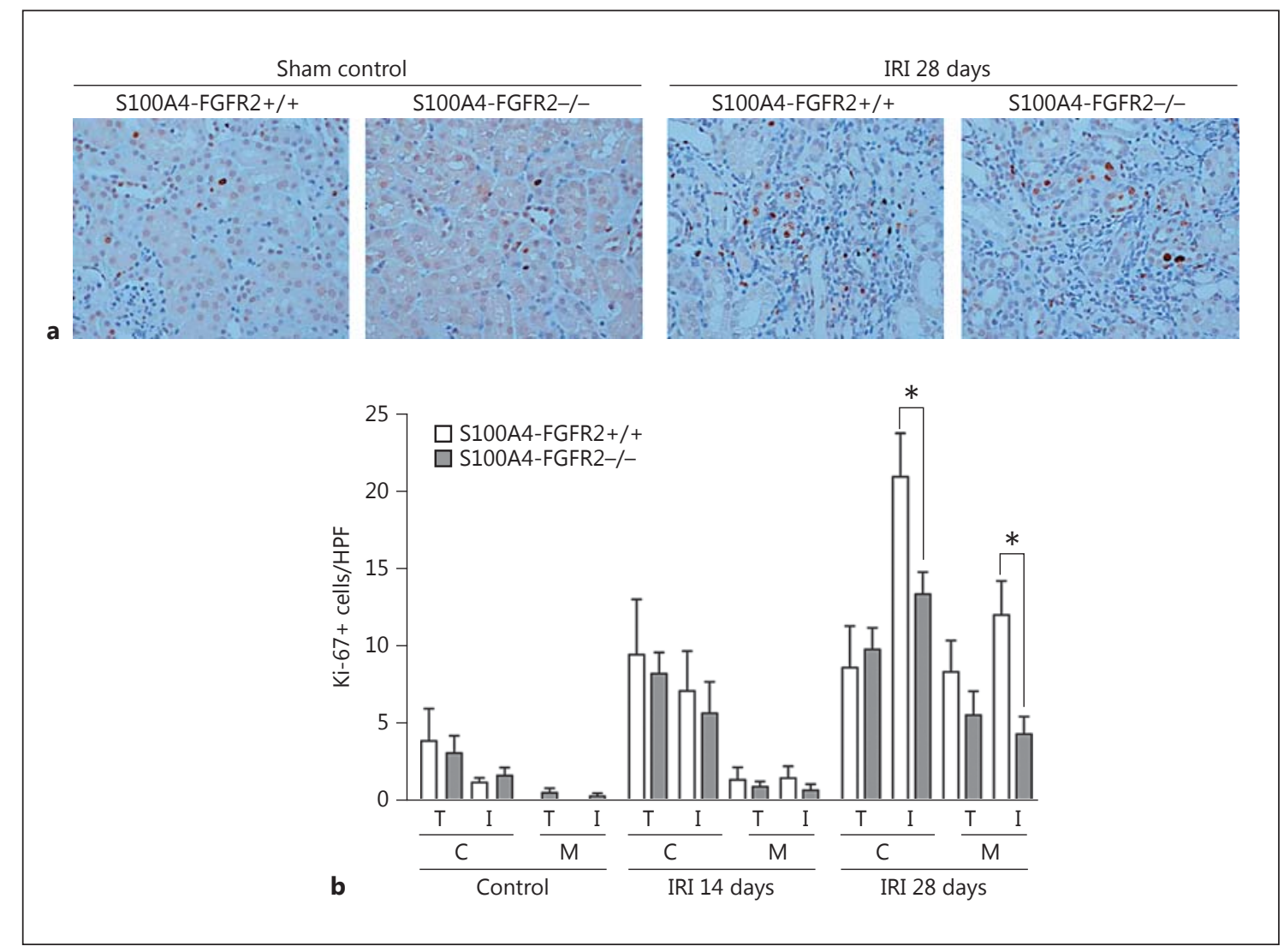

Fig. 4. Interstitial cell proliferation was diminished in the IRI kidneys from S100A4-FGFR2-/- mice. a Representative immunohistochemical staining images showing Ki-67-positive cells in the kidneys from S100A4FGFR2-/- mice and control littermates. b Graph showing quantitative results for Ki-67-positive cells in the kidneys. C, cortical area; M, medullary area; T, tubule; I, interstitium. Data are presented as number of Ki-67-positive cells per HPF $(\times 400){ }^{*} p<0.05$ versus control littermates, $n=4$. FGFR, fibroblast growth factor receptor; HPF, high-power field; IRI, ischemia/reperfusion injury.

Alpha-SMA and FN Expression Were Downregulated in the IRI Kidneys from S100A4-FGFR2-/- Mice

Alpha-SMA expression represents the activation of myofibroblasts in fibrotic tissue. As shown in Figure 3a and $b$, both Western blot analysis and immunofluorescent staining results revealed that $\alpha$-SMA protein expression was largely induced, which was remarkably downregulated in the knockout kidneys 28 days after IRI. In addition, deletion of FGFR2 in fibroblasts also reduced the abundance of FN, a major interstitial matrix protein, in the IRI kidneys. Quantitative analysis of the protein abundance for $\alpha$-SMA and FN in the whole kidney lysate 28 days after IRI is presented in Figure $3 \mathrm{c}$ and $\mathrm{d}$. The data above suggest that the synthesis of ECM as well as the amount of myofibroblasts were reduced in the S100A4FGFR2-/- kidneys 28 days after IRI.
Interstitial Cell Proliferation Was Diminished in the IRI Kidneys from S100A4-FGFR2-/- Mice

The accumulation of interstitial myofibroblasts is mainly determined by their proliferation as well as depletion through cell death. We first detected cell proliferation in the kidneys by immunostaining with anti-Ki-67, a well-used marker of cell proliferation. As shown in Figure 4, Ki-67-positive cells were found in both tubular and interstitial cells of fibrotic kidneys at 28 days after IRI in both S100A4-FGFR2-/- mice and control littermates. The number of proliferative interstitial cells in knockouts was significantly reduced in both the cortical and medullary areas compared to those in the control littermates, while no difference was found as to the Ki-67-positive tubular cells between the two groups either at 14 or 28 days after IRI. These data suggest that loss of FGFR2 in 


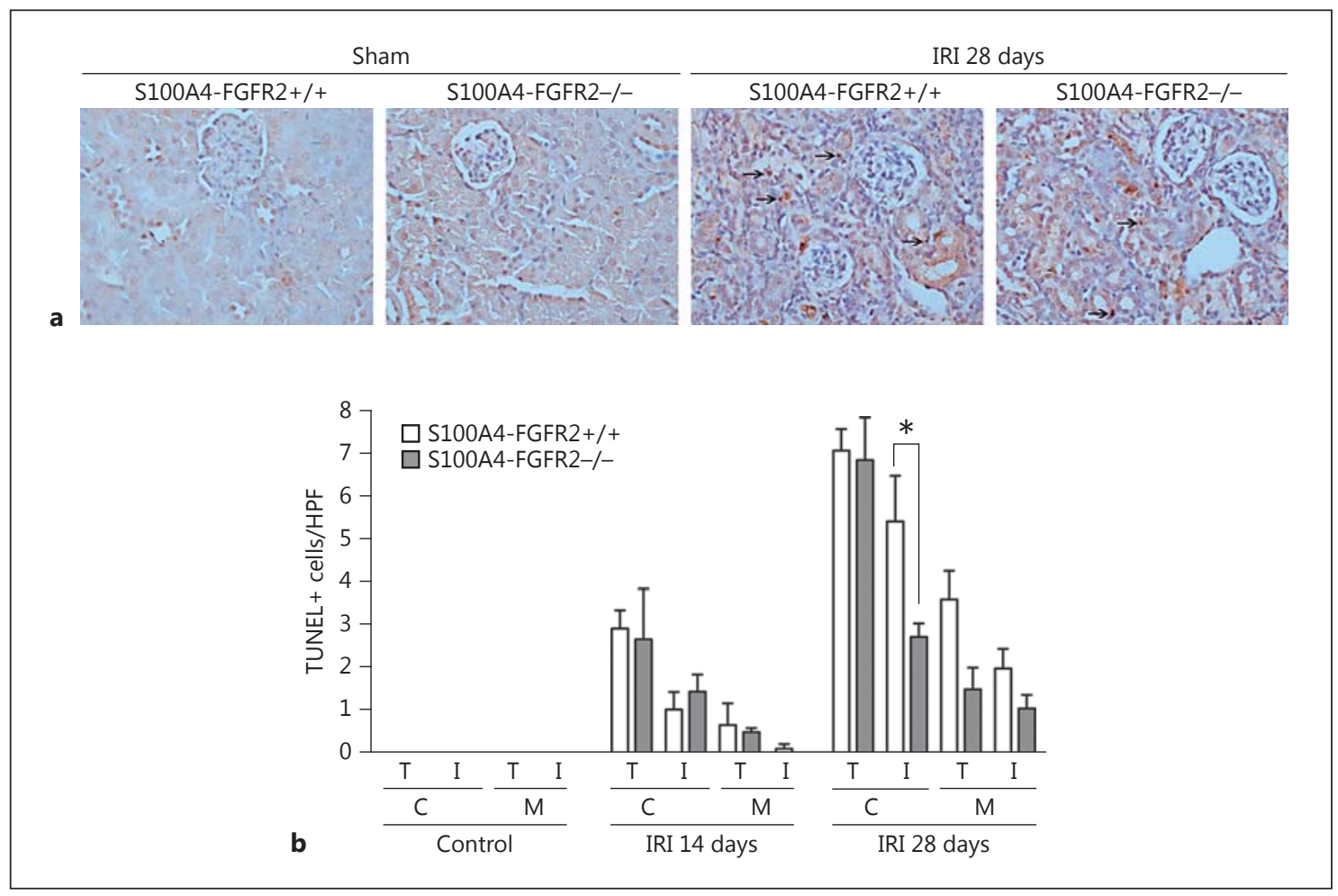

Fig. 5. Interstitial cell apoptosis was less in mice with fibroblast-specific ablation of FGFR2 after IRI. a Representative micrographs showing TUNEL staining for apoptotic cells in the kidneys from S100A4-FGFR2-/- mice and control littermates. Arrows indicate apoptotic cells in the kidneys. b Graph showing the quantitative results for TUNEL-positive cells in the kidneys. C, cortical area; M, medullary area; T, tubule; I, interstitium. Data are presented as TUNEL-positive cell number per HPF $(\times 400) .{ }^{*} p<0.05$ versus control littermates, $n=4$. FGFR, fibroblast growth factor receptor; HPF, high-power field; IRI, ischemia/reperfusion injury.

fibroblasts can significantly inhibit interstitial cell proliferation in the kidney after IRI.

Interstitial Cell Apoptosis Was Less in the IRI Kidneys from S100A4-FGFR2-/- Mice

To further investigate interstitial cell apoptosis in the kidneys after IRI, TUNEL staining was employed on the kidney tissue of both the knockouts and control littermates. As shown in Figure 5, TUNEL staining revealed that both tubular and interstitial cell apoptosis were markedly induced in the fibrotic kidneys from control littermates at 28 days after IRI, which were mainly located in the cortical area. In S100A4-FGFR2-/- mice, tubular cell apoptosis was similar, but interstitial cell apoptosis was significantly diminished compared to control littermates. It is concluded that cell death may not account for the lower accumulation of interstitial myofibroblasts in the knockout kidneys after IRI.

FGFR2 in Kidney Fibrosis
Erk1/2 Phosphorylation Was Less in the Kidneys from S100A4-FGFR2-/- Kidneys after IRI

Erk1/2, one of the major downstream mediators for FGF/FGFR2 signaling, has been reported to be widely involved in renal fibrosis and chronic kidney injury. In this study, Erk1/2 phosphorylation was detected in the IRI kidneys. As shown in Figure 6a and b, Western blot analysis showed that Erk1/2 phosphorylation was markedly decreased in the kidney lysate from the knockouts compared to control littermates at both 14 and 28 days after IRI. Immunofluorescent staining showed the obvious induction and colocalization for $\mathrm{p}$-Erk $1 / 2$ and $a$-SMA in the kidneys from control littermates 28 days after IRI, suggesting the activation of Erk1/2 signaling in the myofibroblasts after IRI, while in the knockout kidneys, less Erk1/2 phosphorylation could be detected (Fig. 6c). These results suggest that deletion of FGFR2 in fibroblast inhibits the activation of Erk1/2 signaling in myofibroblasts from the kidneys after IRI. 


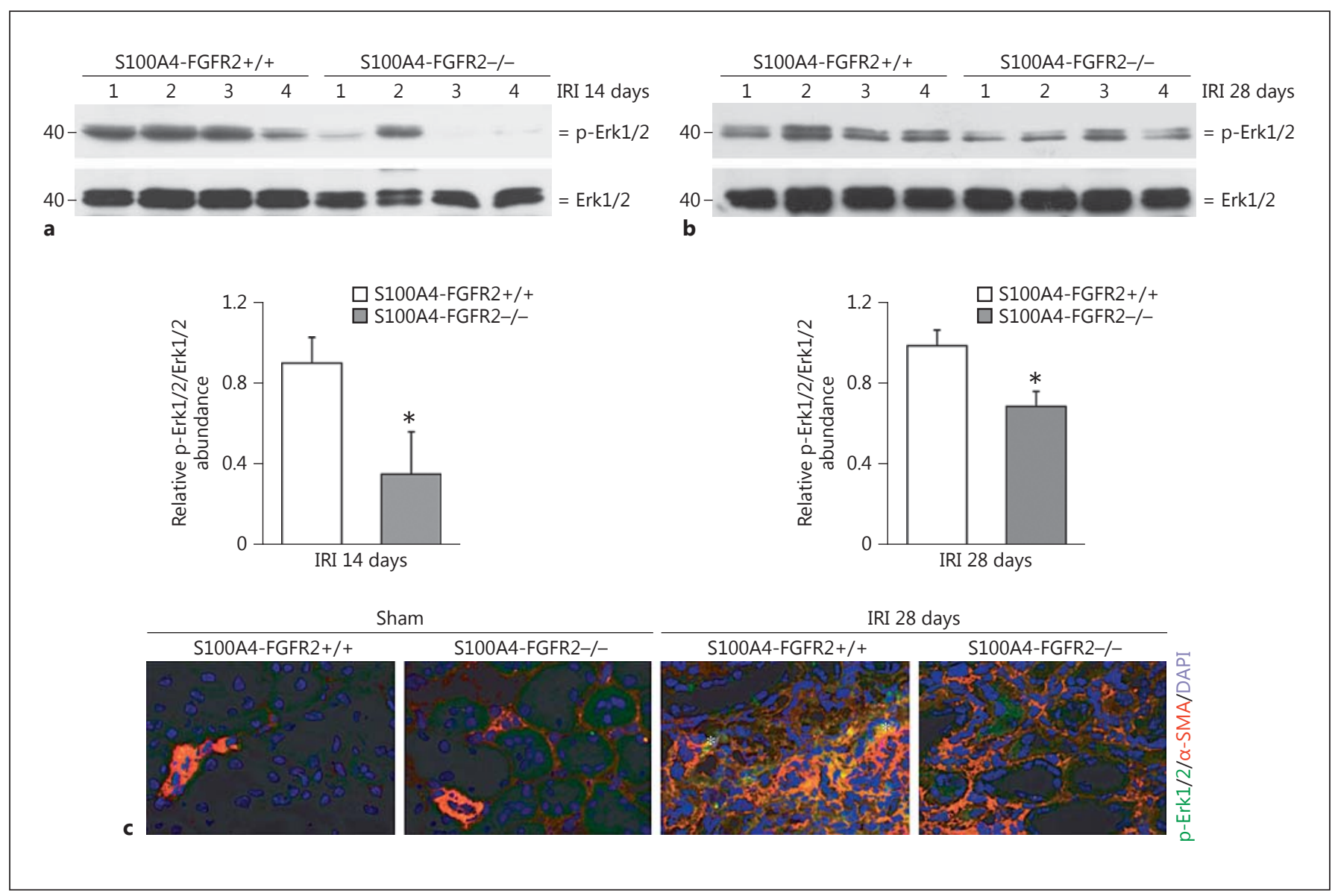

Fig. 6. Erk1/2 phosphorylation was less in the kidneys from S100A4-FGFR2-/- mice after IRI. a, b Western blot analysis demonstrating the reduction in Erk1/2 phosphorylation in S100A4FGFR2-/ - kidneys at 14 days (a) and 28 days (b) after IRI compared to their control littermates. Numbers indicate individual animals. The gels were run under the same experimental conditions. The graphs show the semiquantitative results for $\mathrm{p}$-Erk1/2 in the kidney lysates from S100A4-FGFR2-/- mice and control

\section{Discussion}

We report here that specific ablation of FGFR2 from fibroblasts ameliorated kidney fibrosis after IRI through inhibiting kidney fibroblast activation and proliferation.

The accumulation of myofibroblasts in kidney interstitium is a key factor for ECM deposition and fibrosis [5, $7,8]$. It has been reported that many cell types, such as resident fibroblasts, fibrocytes, tubular cells, and endothelial cells, may be stimulated and transformed to myofibroblasts under disease condition. Recently, LeBleu et al. [8] reported that about $50 \%$ of myofibroblasts come from local resident fibroblasts through proliferation and littermates at 14 and 28 days after IRI. $* p<0.05$ versus control littermates, $n=4$. c Representative immunohistochemical staining images showing the colocalization of $\alpha$-SMA and $\mathrm{p}$-Erk1/2 protein in fibrotic kidneys at 28 days after IRI. Asterisks indicate cells in the kidneys costaining positive for $\alpha$-SMA and p-Erk1/2. $\alpha$-SMA, alpha smooth muscle actin; Erk1/2, extracellular regulated protein kinase 1/2; FGFR, fibroblast growth factor receptor; IRI, ischemia/ reperfusion injury.

activation, part of them from nonproliferating myofibroblasts derived through differentiation from bone marrow (35\%), while fewer are from the endothelial-to-mesenchymal transition program (10\%) and the epithelial-tomesenchymal transition program (5\%). So, proliferation and activation of resident fibroblasts should be the major source of myofibroblasts and play a major contribution for kidney fibrosis $[6,8,9,34,35]$.

In the kidney, FGFs may promote proliferation in various cell types. For example, in adult rats, FGF2 treatment caused focal segmental glomerulosclerosis partly via stimulating podocyte mitosis $[7,36,37]$. Combination of FGF2 with HIV-1 transactivator of transcription could 
induce the dedifferentiation and proliferation of cultured human podocyte [38]. In addition, administration of FGF7 promoted DNA synthesis in cultured renal proximal tubule epithelial cells [23]. While the role for FGFs in interstitial fibroblast activation and renal fibrosis is still debated, Strutz et al. $[39,40]$ reported that, in human kidneys, FGF2 protein and mRNA expression were robustly upregulated in interstitial and tubular cells in end-stage kidneys, and that FGF2 could promote fibroblasts growth. It could also promote kidney tubular epithelial cell epithelial-mesenchymal transition by stimulating the production of microenvironmental proteases. In adult kidneys, the accumulation of myofibroblasts may be determined by their proliferation, cell death, and cell transition from other cell types $[13,41]$. In this study, we found that a-SMA abundance was largely increased in the fibrotic kidneys after IRI, suggesting the accumulation of myofibroblasts. In addition, the number of Ki-67-positive cells in kidney interstitium was also significantly elevated in the fibrotic kidneys from control littermates, whereas it was significantly reduced in S100A4-FGFR2-/- mice, indicating that interstitial cell proliferation was promoted and FGFR2 signaling may play a critical role for interstitial cell proliferation in the fibrotic kidneys after IRI. Our unpublished data demonstrated that FGFR2 signaling is protective against cisplatin-induced tubular cell death. In this study, the number of cells in kidney interstitium staining positive for TUNEL was decreased in S100A4FGFR2-/- mice at 28 days after IRI compared to those in control littermates. The underlying mechanisms are not clear. We found that both interstitial cell proliferation and apoptosis were increased during the progression of kidney fibrosis, suggesting that both pro-proliferative and pro-apoptotic factors may exist in the fibrotic kidneys. In S100A4-FGFR2-/- mice, kidney fibrosis was less severe than in control littermates, so it is possible that the pro-apoptotic factors may also be less in the knockout kidneys.
MAPK/Erk1/2 has been reported to be widely involved in kidney fibrosis and chronic kidney injury. In cultured fibroblasts, MAPK/Erk1/2 may mediate transforming growth factor $\beta 1$-induced fibroblast activation $[42,43]$. In this study, we found that the phosphorylation of Erk1/2 was reduced in S100A4-FGFR2-/- mice compared to that in the control littermates at 14 and 28 days after IRI, which suggest that Erk1/2 signaling may be responsible for FGF/FGFR2-promoted fibroblast proliferation and activation.

In summary, we demonstrated in this study that FGF/ FGFR2 signaling in fibroblasts plays an important role in promoting fibroblast proliferation and activation, which aggravates renal interstitial fibrosis after IRI.

\section{Acknowledgments}

This work was supported by National Science Foundation of China grants $81570611 / \mathrm{H} 0503$ and $81770675 / \mathrm{H} 0503$ and by Science Foundation of Jiangsu Province grant BK20140048 to C. Dai.

\section{Statement of Ethics}

All experiments were performed in accordance with the guidelines and regulations of the Animal Experimentation Ethics Committee of Nanjing Medical University. All experimental protocols were approved by the same committee.

\section{Conflict of Interest Statement}

The authors have declared that no competing interest exists.

\section{Author Contributions}

Z. Xu performed the experiments and wrote the manuscript. C. Dai designed, supervised, and revised the article.

\section{References}

1 Ackland P: Prevalence, detection, evaluation and management of chronic kidney disease. BMJ 2014;348:f7688.

2 Coresh J, Selvin E, Stevens LA, Manzi J, Kusek JW, Eggers P, van Lente F, Levey AS: Prevalence of chronic kidney disease in the United States. JAMA 2007;298:2038-2047.

3 Zhang L, Wang F, Wang L, Wang W, Liu B, Liu J, Chen M, He Q, Liao Y, Yu X, Chen N, Zhang JE, Hu Z, Liu F, Hong D, Ma L, Liu H,
Zhou X, Chen J, Pan L, Chen W, Wang W, Li $\mathrm{X}$, Wang $\mathrm{H}$ : Prevalence of chronic kidney disease in China: a cross-sectional survey. Lancet 2012;379:815-822.

4 Hou F, Jiang J, Chen J, Yu X, Zhou Q, Chen P, Mei C, Xiong F, Shi W, Zhou W, Liu X, Sun S, Xie D, Liu J, Zhang P, Yang X, Zhang Y, Zhang Y, Liang X, Zhang Z, Lin Q, Yu Y, Wu S, Xu X: China Collaborative Study on Dialysis: a multi-centers cohort study on cardiovas- cular diseases in patients on maintenance dialysis. BMC Nephrol 2012;13:94.

5 Falke LL, Gholizadeh S, Goldschmeding R, Kok RJ, Nguyen TQ: Diverse origins of the myofibroblast - implications for kidney fibrosis. Nat Rev Nephrol 2015;11:233244.

6 Boor P, Floege J: The renal (myo-)fibroblast: a heterogeneous group of cells. Nephrol Dial Transplant 2012;27:3027-3036. 
7 Chuang PY, Menon MC, He JC: Molecular targets for treatment of kidney fibrosis. J Mol Med (Berl) 2013;91:549-559.

8 LeBleu VS, Taduri G, O'Connell J, Teng Y, Cooke VG, Woda C, Sugimoto H, Kalluri R: Origin and function of myofibroblasts in kidney fibrosis. Nat Med 2013;19:1047-1053.

9 Piera-Velazquez S, Li Z, Jimenez SA: Role of endothelial-mesenchymal transition (EndoMT) in the pathogenesis of fibrotic disorders. Am J Pathol 2011;179:1074-1080.

10 Boor P, Ostendorf T, Floege J: Renal fibrosis: novel insights into mechanisms and therapeutic targets. Nat Rev Nephrol 2010;6:643656.

11 Ding H, Zhou D, Hao S, Zhou L, He W, Nie J, Hou FF, Liu Y: Sonic hedgehog signaling mediates epithelial-mesenchymal communication and promotes renal fibrosis. J Am Soc Nephrol 2012;23:801-813.

12 Ghosh AK, Vaughan DE: PAI-1 in tissue fibrosis. J Cell Physiol 2012;227:493-507.

13 Hu K, Lin L, Tan X, Yang J, Bu G, Mars WM, Liu Y: tPA protects renal interstitial fibroblasts and myofibroblasts from apoptosis. J Am Soc Nephrol 2008;19:503-514.

14 Jiang L, Xu L, Mao J, Li J, Fang L, Zhou Y, Liu W, He W, Zhao AZ, Yang J, Dai C: Rheb/ mTORC1 signaling promotes kidney fibroblast activation and fibrosis. J Am Soc Nephrol 2013;24:1114-1126.

15 Goetz R, Mohammadi M: Exploring mechanisms of FGF signalling through the lens of structural biology. Nat Rev Mol Cell Biol 2013;14:166-180.

16 Degirolamo C, Sabba C, Moschetta A: Therapeutic potential of the endocrine fibroblast growth factors FGF19, FGF21 and FGF23. Nat Rev Drug Discov 2016;15:51-69.

17 Itoh N, Ornitz DM: Fibroblast growth factors: from molecular evolution to roles in development, metabolism and disease. J Biochem 2011;149:121-130.

18 Liang G, Chen G, Wei X, Zhao Y, Li X: Small molecule inhibition of fibroblast growth factor receptors in cancer. Cytokine Growth Factor Rev 2013;24:467-475.

19 Walker KA, Sims-Lucas S, Bates CM: Fibroblast growth factor receptor signaling in kidney and lower urinary tract development. Pediatr Nephrol 2016;31:885-895.

20 Cancilla B, Ford-Perriss MD, Bertram JF: Expression and localization of fibroblast growth factors and fibroblast growth factor receptors in the developing rat kidney. Kidney Int 1999; 56:2025-2039.
21 Orr-Urtreger A, Givol D, Yayon A, Yarden Y, Lonai P: Developmental expression of two murine fibroblast growth factor receptors, flg and bek. Development 1991;113:1419-1434.

22 Poladia DP, Kish K, Kutay B, Hains D, Kegg $\mathrm{H}$, Zhao H, Bates CM: Role of fibroblast growth factor receptors 1 and 2 in the metanephric mesenchyme. Dev Biol 2006;291: 325-339.

23 Zhao H, Kegg H, Grady S, Truong HT, Robinson ML, Baum M, Bates CM: Role of fibroblast growth factor receptors 1 and 2 in the ureteric bud. Dev Biol 2004;276:403-415.

24 Cui Y, Li Q: Effect of mammogenic hormones on the expression of FGF7, FGF10 and their receptor in mouse mammary gland. Sci China C Life Sci 2008;51:711-717.

25 Yonei-Tamura S, Endo T, Yajima H, Ohuchi H, Ide H, Tamura K: FGF7 and FGF10 directly induce the apical ectodermal ridge in chick embryos. Dev Biol 1999;211:133-143.

26 Ohuchi H, Hori Y, Yamasaki M, Harada H, Sekine K, Kato S, Itoh N: FGF10 acts as a major ligand for FGF receptor 2 IIIb in mouse multi-organ development. Biochem Biophys Res Commun 2000;277:643-649.

27 Sun X, Meyers EN, Lewandoski M, Martin GR: Targeted disruption of Fgf8 causes failure of cell migration in the gastrulating mouse embryo. Genes Dev 1999;13:1834-1846.

28 Walker KA, Sims-Lucas S, Di Giovanni VE, Schaefer C, Sunseri WM, Novitskaya T, de Caestecker MP, Chen F, Bates CM: Deletion of fibroblast growth factor receptor 2 from the peri-wolffian duct stroma leads to ureteric induction abnormalities and vesicoureteral reflux. PLoS One 2013;8:e56062.

29 Floege J, Kriz W, Schulze M, Susani M, Kerjaschki D, Mooney A, Couser WG, Koch KM: Basic fibroblast growth factor augments podocyte injury and induces glomerulosclerosis in rats with experimental membranous nephropathy. J Clin Invest 1995;96:28092819.

30 Strutz F: The role of FGF-2 in renal fibrogenesis. Front Biosci (Schol Ed) 2009;1:125-131.

31 Girshovich A, Vinsonneau C, Perez J, Vandermeersch S, Verpont MC, Placier S, Jouanneau C, Letavernier E, Baud L, Haymann JP: Ureteral obstruction promotes proliferation and differentiation of the renal urothelium into a bladder-like phenotype. Kidney Int 2012;82:428-435.
32 Tsutsumi R, Xie C, Wei X, Zhang M, Zhang X, Flick LM, Schwarz EM, O’Keefe RJ: PGE2 signaling through the EP4 receptor on fibroblasts upregulates RANKL and stimulates osteolysis. J Bone Miner Res 2009;24:17531762 .

33 Lopez-De Leon A, Rojkind M: A simple micromethod for collagen and total protein determination in formalin-fixed paraffin-embedded sections. J Histochem Cytochem 1985;33:737-743.

34 Barnes JL, Gorin Y: Myofibroblast differentiation during fibrosis: role of $\mathrm{NAD}(\mathrm{P}) \mathrm{H}$ oxidases. Kidney Int 2011;79:944-956.

35 Bonventre JV: Can we target tubular damage to prevent renal function decline in diabetes? Semin Nephrol 2012;32:452-462.

36 Kriz W, Hahnel B, Rosener S, Elger M: Longterm treatment of rats with FGF-2 results in focal segmental glomerulosclerosis. Kidney Int 1995;48:1435-1450.

37 Liapis H, Romagnani P, Anders HJ: New insights into the pathology of podocyte loss: mitotic catastrophe. Am J Pathol 2013;183: 1364-1374.

38 Xie X, Colberg-Poley AM, Das JR, Li J, Zhang A, Tang P, Jerebtsova M, Gutkind JS, Ray PE: The basic domain of HIV-tat transactivating protein is essential for its targeting to lipid rafts and regulating fibroblast growth factor-2 signaling in podocytes isolated from children with HIV-1-associated nephropathy. J Am Soc Nephrol 2014;25:1800-1813.

39 Strutz F, Zeisberg M, Hemmerlein B, Sattler B, Hummel K, Becker V, Muller GA: Basic fibroblast growth factor expression is increased in human renal fibrogenesis and may mediate autocrine fibroblast proliferation. Kidney Int 2000;57:1521-1538.

40 Strutz F, Zeisberg M, Ziyadeh FN, Yang CQ, Kalluri R, Muller GA, Neilson EG: Role of basic fibroblast growth factor-2 in epithelialmesenchymal transformation. Kidney Int 2002;61:1714-1728.

41 Zhou D, Tan RJ, Zhou L, Li Y, Liu Y: Kidney tubular $\beta$-catenin signaling controls interstitial fibroblast fate via epithelial-mesenchymal communication. Sci Rep 2013;3:1878.

42 Lan HY: Diverse roles of TGF- $\beta / S$ mads in renal fibrosis and inflammation. Int J Biol Sci 2011;7:1056-1067.

43 Xiao L, Du Y, Shen Y, He Y, Zhao H, Li Z: TGF-beta 1 induced fibroblast proliferation is mediated by the FGF-2/ERK pathway. Front Biosci (Landmark Ed) 2012;17:2667-2674. 